\title{
ATIVIDADE E ESPECIAÇÃO QUÍMICA NA SOLUÇÃO AFETADAS PELA ADIÇÃO DE FÓSFORO EM LATOSSOLO SOB PLANTIO DIRETO EM DIFERENTES CONDIÇÕES DE ACIDEZ ${ }^{(1)}$
}

\author{
Antonio Nolla ${ }^{(2)} \&$ Ibanor Anghinoni ${ }^{(3)}$
}

\begin{abstract}
RESUMO
No sistema plantio direto (SPD), ocorre acúmulo superficial de resíduos de culturas e de nutrientes, especialmente $\mathrm{Pe} C \mathrm{Ca}$, que contribuem para a inativação do $\mathrm{Al}^{3+}$ em solução. Assim, a presença desse elemento no solo não indica, necessariamente, que ocorre fitotoxidez, visto que ânions orgânicos e inorgânicos alteram a dinâmica do Al livre em solução, reduzindo sua atividade. Nestas condições, o conhecimento da atividade e da especiação iônica em solução pode ser útil para o entendimento da sua dinâmica no solo. No presente trabalho, determinaram-se a atividade e a especiação de íons na solução de um Latossolo, cultivado, há sete anos, no sistema plantio direto em diferentes condições de acidez provocadas pela adição anterior (1994) de doses de calcário. Feita a caracterização química do solo do experimento, amostras indeformadas foram coletadas em colunas de PVC rígido, onde se aplicaram, na superfície: $0,40,80,160,320,640$, 1.280 e $2.560 \mathrm{mg} \mathrm{L}^{-1}$ de $\mathrm{P}$ em solução, com posterior incubação, por 30 dias. Extraiuse a solução do solo por centrifugação, determinando-se os atributos de acidez, os cátions e os ânions para estimar a atividade e especiação iônica em solução, utilizando o programa Visual Minteq A2. Com a aplicação das doses de P, houve decréscimo no teor de $\mathrm{Al}$ no solo e na sua atividade em solução. $\mathrm{O}$ aumento de $\mathrm{P}$ na solução não afetou, de forma direta, a inativação de $\mathrm{Al}$, uma vez que não houve formação de fosfatos de Al. No entanto, os ânions orgânicos e inorgânicos, deslocados do complexo de troca pelo fosfato, foram efetivos na complexação do $\mathrm{Al}$ em solução.
\end{abstract}

Termos de indexação: complexação Al-ligantes orgânicos, adubação fosfatada, solução do solo, especiação iônica, amostras indeformadas.

\footnotetext{
(1) Parte da Tese de Doutorado do primeiro autor, apresentada ao Programa de Pós-graduação em Ciência do Solo, Universidade Federal do Rio Grande do Sul - UFRGS. Executada com auxílio do CNPq-PRONEX. Recebido para publicação em janeiro de 2005 e aprovado em outubro de 2006.

(2) Professor Adjunto do Departamento de Agronomia, Universidade Estadual de Maringá - UEM. Rua Goiás 2970, Jardim dos Príncipes, CEP 87502-030 Umuarama (PR). E-mail: nolla73@hotmail.com

(3) Professor Adjunto do Departamento de Solos, Universidade Federal do Rio Grande do Sul - UFRGS. Av. Bento Gonçalves 7712, Caixa Postal 776, CEP 91540-000 Porto Alegre (RS). Bolsista do CNPq. E-mail: ibanghi@ufrgs.br
} 


\title{
SUMMARY: ACTIVITY AND CHEMICAL SPECIATION OF SOIL SOLUTION OFANOXISOL UNDER NO-TILLAGE WITH DIFFERENTACIDITY CONDITIONS AS AFFECTED BY PHOSPHORUS ADDITION
}

\begin{abstract}
In no-tillage systems, crop residues and nutrients, namely phosphorus and calcium, accumulate in soil surface and can inactivate soil aluminum. In this way, only the presence of this element in soil analysis does not indicate plant toxicity, since organic and inorganic anions may affect the free aluminum dynamics by decreasing its activity in the soil solution. Knowledge on ion activity and speciation in soil solution is therefore useful to understand Al dynamics. Ionic activity and speciation were determined in the soil solution of an Oxisol (Rhodic Hapludox), under no-tillage for the seven preceding years and different acidity conditions due to previous applications of variable lime rates in 1994. After the chemical characterization of the study soil, undisturbed samples were collected in PVC columns and subjected to surface application of different phosphorus rates $(0,40,80,160,320,640$, 1.280 , and $2.560 \mathrm{mg} \mathrm{L}^{-1}$ of $P$ in solution) and then incubated for 30 days. Soil solution was directly extracted from the tubes by centrifugation and acidity attributes and cations and anions were determined to estimate ionic activity and speciation using a Visual Minteq A2 program. A decrease in aluminum content and activity in soil solution after phosphorus application was observed. The increase of phosphorus in soil solution did not directly affect aluminum inactivation, because no aluminum phosphates were formed. The increase of phosphorus in soil solution, however, displaced organic and inorganic anions that were effective in aluminum complexation in soil solution.
\end{abstract}

Index terms: aluminum-organic ligand complexation, anion exchange, soil solution, ionic speciation, undisturbed samples.

\section{INTRODUÇÃO}

A aplicação superficial de calcário e de adubos, o não-revolvimento do solo e a manutenção do solo constantemente coberto por plantas ou seus resíduos alteram a dinâmica de nutrientes, especialmente de $\mathrm{Pe}$ de $\mathrm{Ca}$, provocando aumento nas suas concentrações na camada superficial do solo. Além disso, o aumento de $\mathrm{pH}$ do solo, pela calagem, aumenta as cargas negativas e diminui as positivas no complexo de troca e diminui a solubilidade de compostos de $\mathrm{Al}$ e de $\mathrm{Fe}$, aumentando a concentração de P na solução do solo (Ernani et al., 1996; 2000).

A adição de fertilizantes fosfatados em solos sob plantio direto promove a saturação dos sítios de adsorção de $\mathrm{P}$, razão pela qual o $\mathrm{P}$ permanece na forma disponível por mais tempo (Rheinheimer et al., 2003). Este aumento de $\mathrm{P}$ no solo pode também resultar na formação de compostos de baixa solubilidade $\left[\mathrm{AlPO}_{4}\right.$; $\left.\mathrm{Al}(\mathrm{OH})_{2} \mathrm{H}_{2} \mathrm{PO}_{4}\right]$ que precipitam (Raij, 1991; Novais \& Smyth, 1999), de acordo com sua atividade química (Haynes, 1984) e, assim, contribui para a inativação de parte do $\mathrm{Al}^{3+}$ em solução, reduzindo a sua toxidez para as plantas (Wright et al., 1991).

O decréscimo no teor de $\mathrm{Al}$ trocável (de 3,6 para $2,4 \mathrm{cmol}_{\mathrm{c}} \mathrm{dm}^{-3}$ ) e na sua saturação no complexo de troca (de 76 para $44 \%$ ) foi observada por Nolla (2003) pela aplicação superficial em colunas do solo, de 10 x $15 \mathrm{~cm}$, do equivalente a $80 \mathrm{~kg} \mathrm{ha}^{-1} \mathrm{de}_{2} \mathrm{O}_{5}$ em Latossolo Vermelho típico muito ácido sob plantio direto. Esta pode ser uma das razões da baixa ou, mesmo, da falta de resposta à calagem no sistema plantio direto em solos com $\mathrm{pH}$ em água baixo (em torno de 5,0) com P disponível na classe alto e muito alto (Anghinoni \& Salet, 2000). O maior teor de Ca trocável na camada superficial do solo nesse sistema de cultivo pode também diminuir o efeito fitotóxico de Al por sua ação desintoxicante (Marschner, 1995), mas, pode, potencialmente, retirar $\mathrm{P}$ da solução pela formação de fosfatos de $\mathrm{Ca}$.

Os ácidos orgânicos, que se acumulam na superfície do solo sob sistema plantio direto, podem contribuir efetivamente na complexação de grande parte do Al em solução (Anghinoni \& Salet, 1998; Salet et al., 1999). Segundo Ritchie et al. (1988), a complexação do Al com os ligantes orgânicos geralmente é mais forte do que a complexação com ligantes inorgânicos. Estes últimos podem exercer grande influência na redução da toxidez do $\mathrm{Al}$, especialmente em $\mathrm{pH} \leq 5,5$ (Shoji \& Fujiwara, 1984) com o Al ligando-se à hidroxila, ao fluoreto, ao sulfato e ao fosfato (Sposito, 1989).

A dinâmica dos íons no solo tem sido estudada com base na determinação de cátions e ânions na sua solução. Portanto, não é possível identificar as condições em que ocorre a fitotoxidez por $\mathrm{Al}$, especialmente no sistema plantio direto, por não haver separação entre as espécies tóxicas das chamadas nãotóxicas, que podem estar complexadas com ligantes inorgânicos e, ou, orgânicos (Alva et al., 1986; Hue et al., 1986). Nesta, os íons ocorrem sob diferentes formas graças às reações e interações, que reduzem $o$ 
potencial químico dos íons livres. Assim, parte do Al na solução do solo pode ser complexado, polimerizado ou precipitado por ânions orgânicos (radicais orgânicos $\left.-\mathrm{O}^{-}, \mathrm{COO}^{-}\right)$e inorgânicos $\left(\mathrm{OH}^{-}, \mathrm{PO}_{4}^{3-}, \mathrm{SO}_{4}{ }^{2-}\right.$ e F- $)$, alterando suas espécies químicas e reduzindo sua atividade (Hue et al., 1986).

Assim, o aumento da concentração de $\mathrm{P}$ na solução do solo, seja pela adubação, seja pelo não-revolvimento do solo no sistema plantio direto, seja pelo uso de calcário, que eleva o $\mathrm{pH}$, pode reduzir o efeito tóxico do $\mathrm{Al}$, tanto pela complexação em solução, como por sua precipitação.

Os objetivos deste trabalho foram estudar a retenção de $\mathrm{P}$ adicionado ao solo e a dinâmica de íons na solução de um Latossolo com diferentes níveis de acidez, cultivado no sistema plantio direto, visando identificar quais os componentes e os mecanismos responsáveis pela redução da atividade do $\mathrm{Al}$ em solução.

\section{MATERIAL E MÉTODOS}

Foi realizado um ensaio de incubação, em laboratório, de solo em colunas indeformadas provenientes de um experimento de campo, instalado pela Embrapa Trigo, em um Latossolo Vermelho distrófico típico com 53 \% de argila, no município de Passo Fundo (RS). O local era uma área de campo nativo até 1988 , quando passou a ser cultivado no sistema convencional, até 1994, com milho (Zea mays) ou soja (Glycine max), no verão, e aveia preta (Avena strigosa), no inverno. Nesse ano, o experimento foi instalado no sistema plantio direto, com a aplicação de três doses de calcário: 1,0 (1/24 SMP), 6,0 (1/4 SMP) e 24,0 t ha-1 (1 SMP - pH 6,0), num delineamento em blocos casualizados, com quatro repetições. A área foi cultivada até à coleta do solo para o presente trabalho na sucessão aveia preta/soja/trigo(Triticum vulgare)/ milho, recebendo a adubação de $\mathrm{N}, \mathrm{P}$ e K indicada pela Comissão de Fertilidade do Solo (CFS RS/SC, 1989; 1995), com os adubos aplicados a lanço. O rendimento de grãos da soja nas parcelas de campo (safra 2000/ 2001) foi de 1,51, 3,67 e 4,49 $\mathrm{tha}^{-1}$, quando foram aplicados (1994) 0, 6,0 e 24,0 t ha-1 de calcário, respectivamente.

Para o ensaio, amostras indeformadas, em colunas de PVC de 5 (diâmetro) x $10 \mathrm{~cm}$ de solo (altura), totalizando oito colunas por parcela, foram coletadas no experimento e submetidas à incubação com oito doses de P. No laboratório, aplicaram-se superficialmente, nas colunas, $0,40,80,160,320,640,1.280 \mathrm{e}$ $2.560 \mathrm{mg} \mathrm{L}^{-1}$ de $\mathrm{P}$ na forma de $\mathrm{KH}_{2} \mathrm{PO}_{4}$ em solução. As colunas, com o fundo coberto por papel filtro Wattman 42, para evitar a perda de solo e água, foram, então, colocadas em copos plásticos para incubar, durante 30 dias, em câmara de crescimento, com temperatura entre 24 e $27^{\circ} \mathrm{C}$, mantendo-se a umidade do solo a, aproximadamente, $90 \%$ da capacidade de campo (determinada na tensão de 0,35 atm com valor $\left.\theta=0,39 \mathrm{~m}^{-3} \mathrm{~m}^{-3}\right)$.

Após a incubação, as colunas, com a aplicação das diferentes doses de $\mathrm{P}$, foram centrifugadas, por $30 \mathrm{~min}$, a $2.450 \mathrm{rpm}$ (centrífuga Janetzki K-60), para a extração da solução do solo. Após a retirada de uma alíquota para a determinação do $\mathrm{pH}$ e $\mathrm{CO}$ solúvel (Moore, 1985), o restante da solução do solo de cada tratamento foi mantido em baixa temperatura $\left(-18^{\circ} \mathrm{C}\right)$, para posterior determinação das concentrações dos cátions e ânions da solução.

No solo, foram avaliados: $\mathrm{CO}$ total, conforme método de Walkley \& Black; $\mathrm{pH}-\mathrm{H}_{2} \mathrm{O}$ e $\mathrm{pH}-\mathrm{CaCl}_{2}$; $\mathrm{Ca}, \mathrm{Mg}$ e $\mathrm{Al}$ trocáveis $\left(\mathrm{KCl} 1 \mathrm{~mol} \mathrm{~L}^{-1}\right)$, sendo o $\mathrm{Ca}$ e o $\mathrm{Mg}$ determinados por espectrofotômetro de absorção atômica e o Al, por titulação, com NaOH $0,0125 \mathrm{~mol} \mathrm{~L}^{-1}$; o K foi extraído pelo método Mehlich-1 e determinado por fotômetro de chama, todos conforme Tedesco et al. (1995). O P foi extraído pelo método Mehlich-1 e determinado por colorímetro, conforme método de Murphy \& Rilley (1962).

$\mathrm{Na}$ solução do solo, foram determinados: o $\mathrm{pH}$ (potenciômetro com eletrodo de vidro), o $\mathrm{Ca}, \mathrm{o} \mathrm{Mg}$ e o Mn (absorção atômica), o K e o Na (fotômetro de chama) e o $\mathrm{Al}$ (absorção atômica com forno de grafite). O CO solúvel foi determinado segundo Moore (1985); o amônio, pelo método de Franson (1995); e os ânions: fosfato, sulfato, cloreto e nitrato, por cromatografia iônica (Jeffery et al., 1992) por cromatógrafo Dionex - D-120 e coluna ASCHA.

A partir das quantidades adicionadas de $\mathrm{P}$ e dos dados de sua concentração na solução extraída das colunas, foi avaliada a capacidade de retenção de $\mathrm{P}$ afetada pela condição de acidez do solo pela isoterma de Langmuir (Barrow, 1978). A estimativa da atividade e da especiação química dos íons em solução foi efetuada pelo programa Visual Minteq (for Windows) versão 2.12 (Gustaffson, 2002), baseado no programa Minteq A2 versão 4.0.

\section{RESULTADOS E DISCUSSÃO}

O solo apresentava, antes do ensaio de incubação com doses de $\mathrm{P}$ em colunas (após sete anos da aplicação de doses de calcário e do cultivo no sistema plantio direto), grande variação nos atributos de acidez de acordo com as doses de calcário adicionadas em 1994 (Quadro 1). A condição mais ácida caracteriza-se por pH e saturação por bases muito baixos e por teor e saturação por Al muito altos. Os teores de P e K disponíveis (Mehlcih-1) foram classificados (CFS RS/ $\mathrm{SC}, 1995)$ como alto e médio, respectivamente, para o solo em estudo.

A incubação do solo com doses crescentes de P nas colunas resultou em aumento linear no seu teor disponível (Mehlich-1), chegando a atingir, na maior 
dose aplicada (2.560 $\mathrm{mg} \mathrm{dm}^{-3} \mathrm{de} \mathrm{P}$ ), 28 vezes o valor do seu teor inicial (Figura 1a). A cinética de adsorção desse nutriente pela isoterma de Langmuir (Figura 2) evidencia aumento na capacidade de retenção de $\mathrm{P}$ pelo solo à medida que a acidez aumenta. Assim, a capacidade de adsorção máxima aumentou em $14 \mathrm{e}$ $83 \%$ com a diminuição do $\mathrm{pH}$ de 6,4 para 4,5 e 4,0, respectivamente (Quadro 2). Isto indica a menor necessidade de adição de $\mathrm{P}$, quer para promover a sua saturação no solo, quer para atingir o teor crítico para o crescimento das culturas, com a diminuição da acidez do solo, como também verificado por Mendez \& Kamprath (1978) e Ernani \& Barber (1991). Esse aumento na capacidade de retenção relaciona-se com o teor de Al trocável, indicando a possibilidade de formação de fosfatos de $\mathrm{Al}$ precipitados, mas não apresenta relação com o teor de $\mathrm{CO}$, que não foi alterado nas diferentes condições de acidez (Quadro 2). No entanto, a constante relacionada com a energia de ligação aumentou (de 0,014 para $0,022 \mathrm{~L} \mathrm{mg}^{-1}$ ), à medida que se reduziram as condições de acidez do solo (Quadro 2). Provavelmente, isto pode estar ocorrendo porque, segundo Rheinheimer et al. (2003), as isotermas de adsorção não permitem separar os fenômenos responsáveis pelo desaparecimento do $\mathrm{Pem}$ solução, provavelmente em virtude da formação de precipitados.

Concomitantemente à elevação do teor de $\mathrm{P}$ disponível (Mehlich-1 - Figura 1a) pela adição de doses desse nutriente, houve elevação do $\mathrm{pH}$ (Figura 1b) e diminuição dos teores de Al trocável no solo (Figura 1c), nas duas condições de maior acidez ( $\mathrm{pH} 4,0$ e 4,5) do solo. O teor de CO (Quadro 2), por sua vez, não foi afetado pela utilização de doses crescentes de adubo fosfatado (Nolla, 2003). Isto ocorreu, provavelmente, porque, no sistema plantio direto, há acúmulo de matéria orgânica (Lovato, 2001)

Quadro 1 Atributos químicos da camada de 0-20 cm de um Latossolo Vermelho distrófico típico cultivado por sete anos, no sistema plantio direto, após a aplicação de calcário

\begin{tabular}{|c|c|c|c|c|c|c|}
\hline \multirow{2}{*}{ Atributo químico } & \multicolumn{6}{|c|}{ Dose de calcário aplicada em $1994\left(\mathrm{t} \mathrm{ha}^{-1}\right)$} \\
\hline & 1,0 & & 6,0 & & $24,0(1 \mathrm{SMF}$ & pH 6,0) \\
\hline $\mathrm{pH} \mathrm{H}_{2} \mathrm{O}$ & 4,0 & $\mathrm{C}$ & 4,6 & $\mathrm{~b}$ & 6,4 & a \\
\hline $\mathrm{pH} \mathrm{CaCl} 2$ & 3,3 & $\mathrm{C}$ & 4,0 & $\mathrm{~b}$ & 5,7 & $\mathrm{~A}$ \\
\hline pH SMP & 4,5 & $\mathrm{C}$ & 5,2 & $\mathrm{~b}$ & 6,5 & $\mathrm{~A}$ \\
\hline Ca trocável $\left(\mathrm{cmol}_{\mathrm{c}} \mathrm{kg}^{-1}\right)$ & 0,64 & $\mathrm{C}$ & 2,58 & $\mathrm{~b}$ & 6,98 & $\mathrm{~A}$ \\
\hline Mg trocável $\left(\mathrm{cmol}_{\mathrm{c}} \mathrm{kg}^{-1}\right)$ & 0,22 & $\mathrm{C}$ & 1,78 & $\mathrm{~b}$ & 4,64 & $\mathrm{~A}$ \\
\hline $\mathrm{Al}$ trocável $\left(\mathrm{cmol}_{\mathrm{c}} \mathrm{kg}^{-1}\right)$ & 3,44 & $\mathrm{a}$ & 1,33 & $\mathrm{~b}$ & 0,01 & $\mathrm{C}$ \\
\hline K Mehlich-1(mg kg-1) & 55 & a & 49 & a & 46 & $\mathrm{~A}$ \\
\hline Saturação por Al (\%) & 77,5 & $\mathrm{a}$ & 22,9 & $\mathrm{~b}$ & 0,09 & $\mathrm{C}$ \\
\hline P Mehlich-1(mg kg-1) & 27,1 & $\mathrm{a}$ & 26,6 & a & 24,5 & $\mathrm{~A}$ \\
\hline $\mathrm{CO}\left(\mathrm{g} \mathrm{kg}^{-1}\right)$ & 30,0 & $\mathrm{a}$ & 29,2 & a & 29,5 & $\mathrm{~A}$ \\
\hline
\end{tabular}

Médias seguidas pela mesma letra, na linha, não diferem entre si, pelo teste de Tukey $(\mathrm{P}<0,05)$.

Quadro 2. Constantes da isoterma de Langmuir de um Latossolo Vermelho distrófico típico (camada de 0$10 \mathrm{~cm}$ ), com diferentes níveis de acidez do solo nas colunas onde foram aplicadas doses crescentes de fósforo

\begin{tabular}{|c|c|c|c|c|}
\hline \multicolumn{3}{|c|}{ Condição do solo } & \multirow{2}{*}{$\begin{array}{c}\text { Capacidade máxima } \\
\text { de adsorção }\end{array}$} & \multirow{2}{*}{$\begin{array}{c}\text { Constante } \\
\text { relacionada com } \\
\text { energia de ligação }\end{array}$} \\
\hline pH água & Al trocável & C orgânico & & \\
\hline & $\mathrm{cmol}_{\mathrm{c}} \mathrm{kg}^{-1}$ & $\mathrm{~g} \mathrm{~kg}^{-1}$ & $\mathrm{mg} \mathrm{g}^{-1}$ & $\mathrm{~L} \mathrm{mg}^{-1}$ \\
\hline 4,0 & 3,22 & 29,4 & 5,23 & 0,014 \\
\hline 4,5 & 1,41 & 30,4 & 3,26 & 0,022 \\
\hline 6,4 & 0,00 & 30,0 & 2,86 & 0,022 \\
\hline
\end{tabular}


(a)

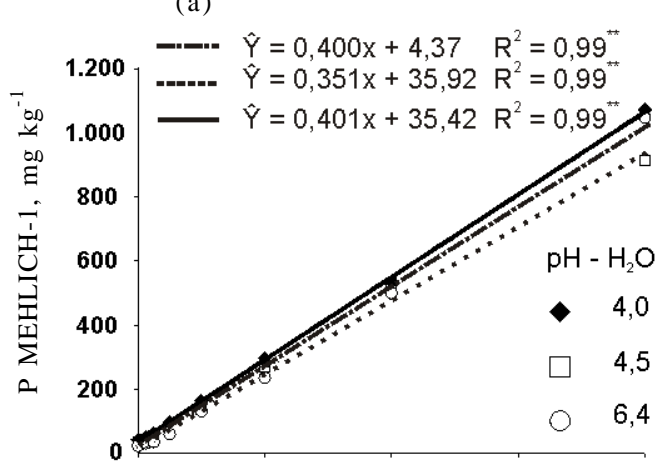

(b)

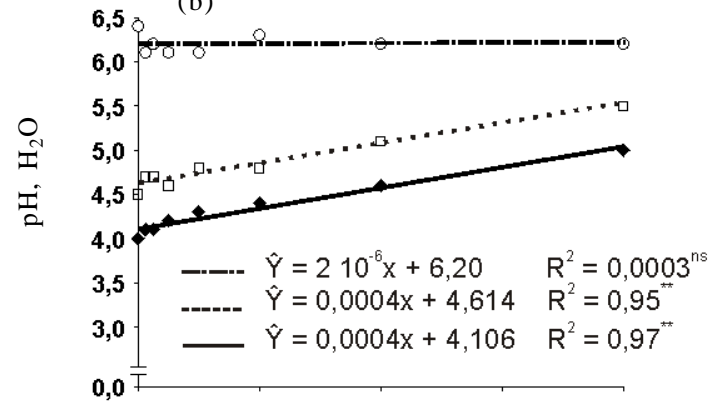

(c)

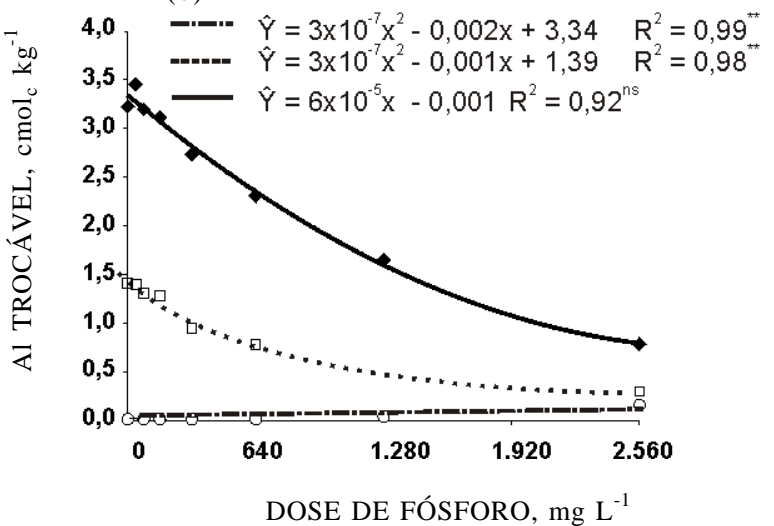

Figura 1. Atributos químicos em colunas de solo afetados pela aplicação de doses de fósforo em diferentes condições de $\mathbf{p H}$ sob plantio direto: fósforo disponível (a); $\mathrm{pH} \mathrm{em} \mathrm{H}_{2} \mathrm{O}$ (b) e alumínio trocável (c).

mais estável, decorrente de sua proteção físicoquímica, proveniente dos efeitos de estruturação e agregação em solo não mobilizado.

O estudo da atividade e da especiação química de íons em solução permite uma avaliação mais detalhada a respeito da eficiência dos componentes e o mecanismo responsável pela neutralização e, ou, complexação do $\mathrm{Al}$ em solução, reduzindo seu efeito fitotóxico no sistema plantio direto. A atividade do íon fosfato na solução do solo (Figura 3a) foi baixa em qualquer nível de acidez do solo nos tratamentos com aplicação até $640 \mathrm{mg} \mathrm{dm}^{-3} \mathrm{de} \mathrm{P.} \mathrm{A} \mathrm{partir} \mathrm{dessa} \mathrm{dose,}$ houve aumento exponencial na sua atividade, provavelmente pela maior saturação dos sítios de adsorção na fase sólida.

Houve efeito da aplicação de $\mathrm{P}$, diminuindo a atividade do $\mathrm{Al}$ na solução (Figura $3 \mathrm{~b}$ ), que foi maior na condição de maior acidez $(\mathrm{pH}=4,0$ e Al trocável = $\left.3,22 \mathrm{cmol}_{\mathrm{c}} \mathrm{kg}^{-1}\right)$. Essa atividade, na maior dose de $\mathrm{P}$ aplicada $\left(2.560 \mathrm{mg} \mathrm{L}^{-1}\right)$ nessa condição de acidez, correspondeu à atividade observada na condição de acidez intermediária ( $\mathrm{pH} 4,5$ e $\mathrm{Al}$ trocável = $\left.1,41 \mathrm{cmol}_{\mathrm{c}} \mathrm{dm}^{-3}\right)$.

O CO dissolvido é capaz de inativar parte do $\mathrm{Al}$ em solução (Salet et al., 1999). Observa-se que a atividade de CO em solução, diferentemente do teor de $\mathrm{C}$ total, que não foi afetado (Quadro 2), aumentou com a aplicação de doses de calcário e de P (Figura 3c), especialmente na condição menos ácida. Esse aumento do $\mathrm{pH}$ deveu-se ao maior crescimento das culturas no campo com conseqüente maior deposição de restos culturais, o que deve ter contribuído para o aumento da atividade do $\mathrm{CO}$ solúvel em solução no pH 3,5 e 6,4 (Figura 3c). Provavelmente, doses elevadas de fosfato deslocaram ânions orgânicos da troca por ação de massa, reduzindo o $\mathrm{Al}$ em solução pela formação de Al-orgânico (Hens \& Merckx, 2002).

A atividade de $\mathrm{Ca}$ (Figura $3 \mathrm{~d}$ ) foi proporcional aos seus teores no solo (Quadro 1), resultantes da aplicação das diferentes doses de calcário no início do experimento (1994). De maneira geral, a atividade desse elemento na solução decresceu com a adição de $\mathrm{P}$, especialmente na condição de menor acidez do solo (pH 6,4 - Figura 3d). Nessa condição, o P acumulado poderia reagir com cátions divalentes, preferencialmente o $\mathrm{Ca}$, favorecendo a formação de precipitados de fosfatos de Ca (Novais \& Smyth, 1999).

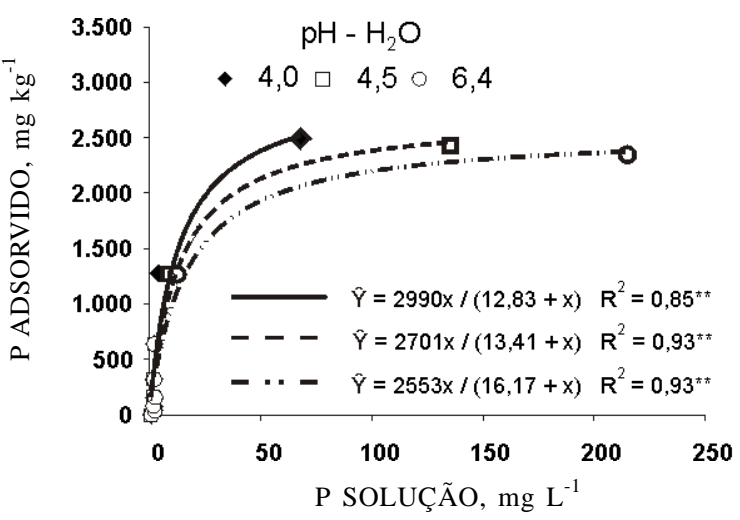

Figura 2. Relação entre a concentração de fósforo na solução do solo e o fósforo adsorvido no complexo de troca de um Latossolo Vermelho distrófico típico (camada de 0-10 cm) em diferentes condições de acidez e submetidas a aplicação de doses de fósforo. 
(a)

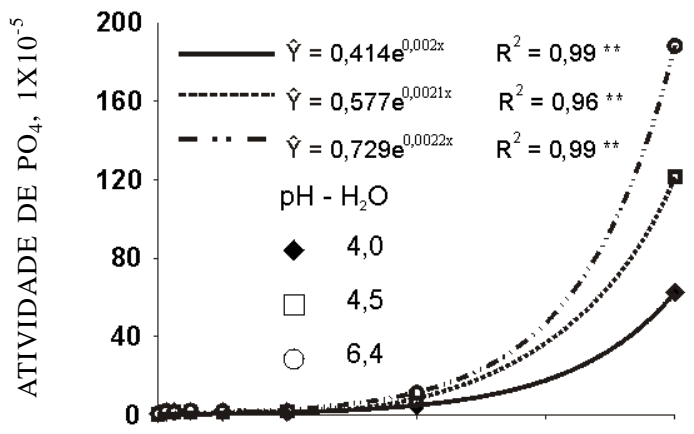

(c)

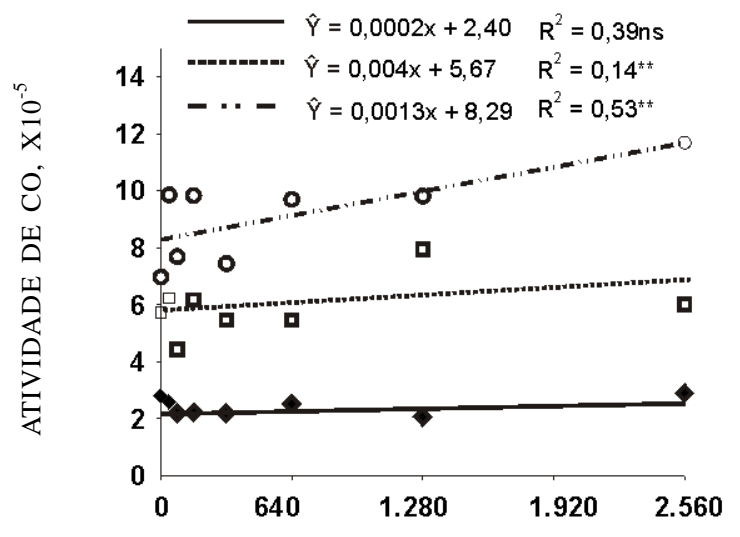

(b)

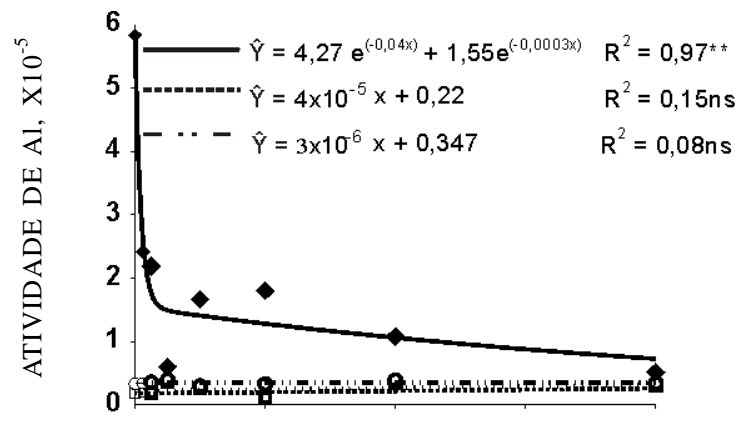

(d)

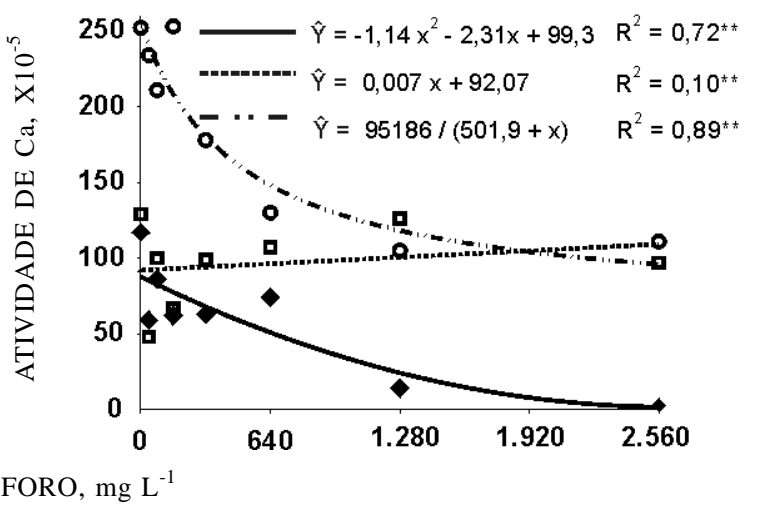

Figura 3. Atividade de fosfato (a), alumínio (b), carbono orgânico (c) e cálcio (d) na solução de um Latossolo Vermelho distrófico típico (camada de 0-10 cm), cultivado, por sete anos, no sistema plantio direto em diferentes condições de acidez e submetidas a aplicação de doses de fósforo.

A espécie predominante de fosfato na solução do solo nas condições mais ácidas (Figura 4a,b), com $90 \%$ ou mais da espécies em solução, foi o $\mathrm{H}_{2} \mathrm{PO}_{4}^{-}$. Esta espécie também predominou, com uma concentração relativa entre 70 e $80 \%$, na condição de menor acidez (Figura 4c), seguida por $\mathrm{HPO}_{4}{ }^{2-}$, com, aproximadamente, $10 \%$. Observa-se, neste tratamento, que, além das duas espécies predominantes, houve formação dos complexos $\mathrm{CaHPO}_{4}, \mathrm{CaH}_{2} \mathrm{PO}_{4}^{+}, \mathrm{MgHPO}_{4}$ e $\mathrm{MgH}_{2} \mathrm{PO}_{4}{ }^{+}$. Isto pode ter acontecido porque, nos tratamentos com $\mathrm{pH}$ de 6,0 ou mais, ocorre concentração mais elevada de Ca e de Mg trocáveis (Quadro 1), que reagem com o fosfato acumulado (Novais \& Smyth, 1999).

Esperava-se a formação de fosfatos de Al, uma vez que aplicação do P reduziu efetivamente a concentração de Al no solo (Figura 1c) e na solução (Figura 3b), principalmente nas condições de maior acidez. Entretanto, isto não ocorreu, mesmo com aplicação de doses elevadas de P (Figura 5). Provavelmente, não houve formação de fosfato de $\mathrm{Al}$, em razão do valor baixo da constante de equilíbrio $(\mathrm{Ke}<-19,5)$, que reflete uma baixa estabilidade e uma baixa afinidade entre o $\mathrm{Al}$ e P nessas condições (Alva \& Sumner, 1989). Esta baixa capacidade de complexação de $\mathrm{Al}$ por fosfato também foi observada por Salet et al. (1999) no sistema plantio direto, onde a concentração relativa da espécie $\mathrm{AlH}_{2} \mathrm{PO}_{4}{ }^{2+}$ foi menor do que $0,1 \%$. Pode-se inferir que, graças à grande capacidade de retenção de $\mathrm{P}$ pelo complexo de troca do solo e à baixa estabilidade dos fosfatos de $\mathrm{Al}$, a ação do $\mathrm{P}$ na neutralização direta do $\mathrm{Al}$ em solução é baixa. Outros componentes, como os ligantes orgânicos e inorgânicos, devem ter sido mais efetivos na diminuição da atividade do $\mathrm{Al}$.

A espécie dominante de $\mathrm{Al}$ em condições de maior acidez foi o $\mathrm{Al}$ livre $\left(\mathrm{Al}^{3+}\right.$ ) (Figura 5a). O Al complexado por ligantes orgânicos (Al-orgânico) também foi elevado nessa condição, com aumento de sua participação com a dose de fosfato aplicada. Isto deveu-se ao aumento de fosfato e de $\mathrm{OH}^{-}$(aumento do $\mathrm{pH}$ - Figura 1b) que deslocaram ânions orgânicos do complexo de troca para a solução (Figura 3c), conforme observado por Hens \& Merckx (2002). Assim, os ânions orgânicos ocasionaram a formação do complexo Al-orgânico em solução (aumento de $17,5 \%$ para $55 \%$ ), reduzindo a concentração relativa do Al livre (Figura 5a). Em condições de acidez intermediária (Figura 5b), a atividade de Al é baixa. Dentre as espécies 


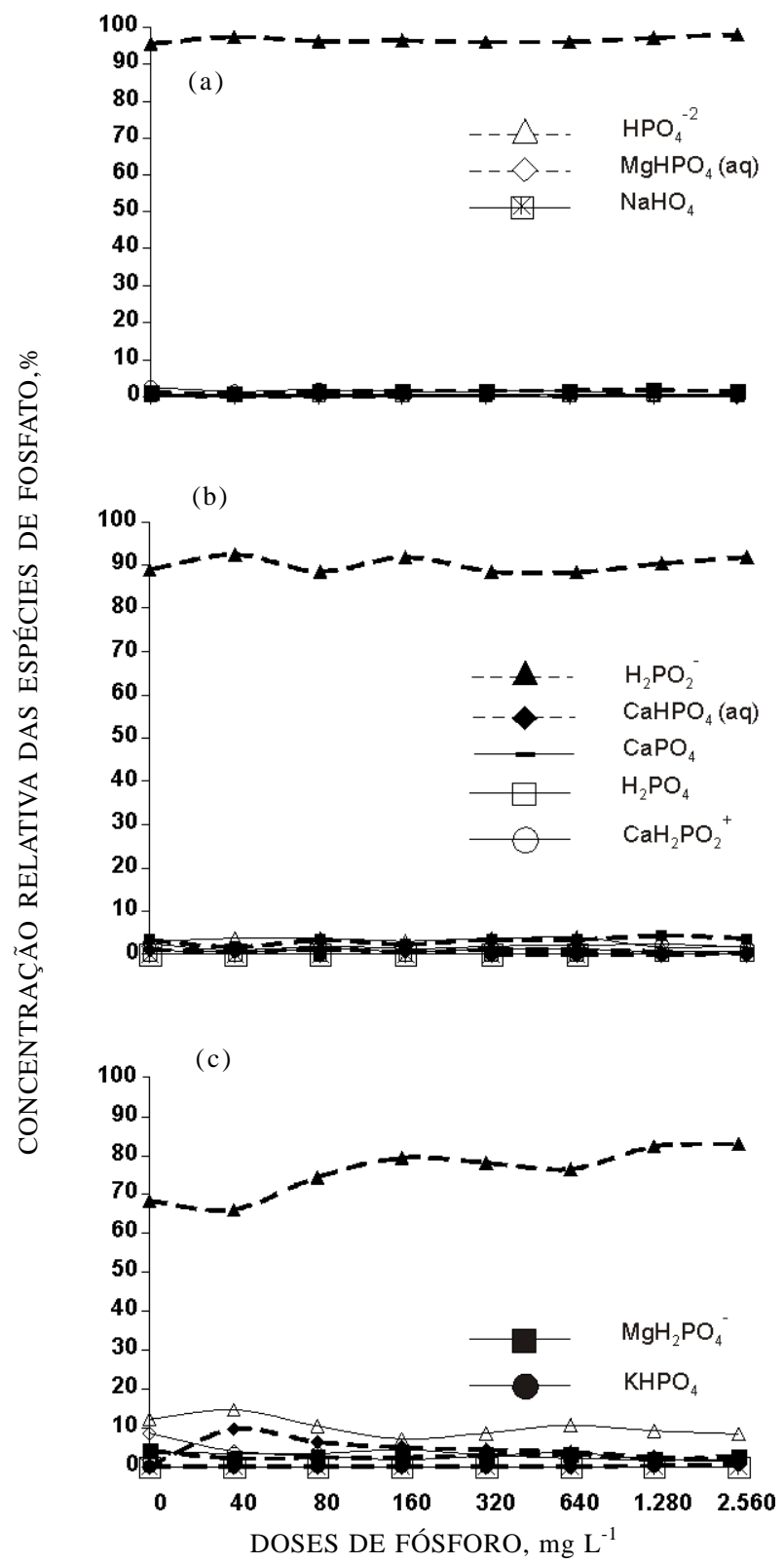

Figura 4. Concentração relativa de espécies de fosfato na solução de um Latossolo Vermelho distrófico típico, cultivado, por sete anos no sistema plantio direto em diferentes condições de acidez: pH 4,0 (a); pH 4,5 (b) e pH 6,4 (c) e submetidas a aplicação de doses de fósforo.

predominantes, destacaram-se o $\mathrm{Al}(\mathrm{OH})_{2}{ }^{+} \mathrm{Al}(\mathrm{OH})^{2+} \mathrm{e}$ o Al-orgânico, que foram importantes na diminuição da atividade do $\mathrm{Al}^{3+}$ em solução. No tratamento com menor acidez $(\mathrm{pH}=6,4$; Figura $5 \mathrm{c})$, predominaram as espécies $\mathrm{Al}(\mathrm{OH})_{2}{ }^{+}$e $\mathrm{Al}(\mathrm{OH})_{4}{ }^{-}$, não-tóxicas para as plantas. A complexação do $\mathrm{Al}$ com os ácidos orgânicos é pouco pronunciada nessa condição de acidez, pela menor reatividade do $\mathrm{Al}$ com os ânions orgânicos em solução em relação à sua reatividade com $\mathrm{OH}^{-}$(Shoji \& Fujiwara, 1984).
Para todas as condições de acidez, a espécie orgânica dominante foi o CO livre (COD livre - Figura 6), provavelmente porque, no sistema plantio direto, a decomposição de restos culturais na camada superficial do solo provoca liberação contínua de CO para a solução do solo, que foi de até $216 \%$ no presente trabalho. Na condição mais ácida (Figura 6a), outra espécie que se destacou foi o $\mathrm{Al}-\mathrm{CO}$ dissolvido (Al-COD), com mais de $40 \%$ no tratamento sem a aplicação de fosfato. No entanto, à medida que as doses de fosfato aumentaram, a concentração relativa do Al-COD diminuiu (Figura 6a). Apesar desta redução, a concentração e a atividade de $\mathrm{Al}$ permaneceram baixas (Figura 5a), porque a adição de altas doses de fosfato, além de aumentar a concentração de ligantes orgânicos (complexação Al-orgânico), aumentou o pH

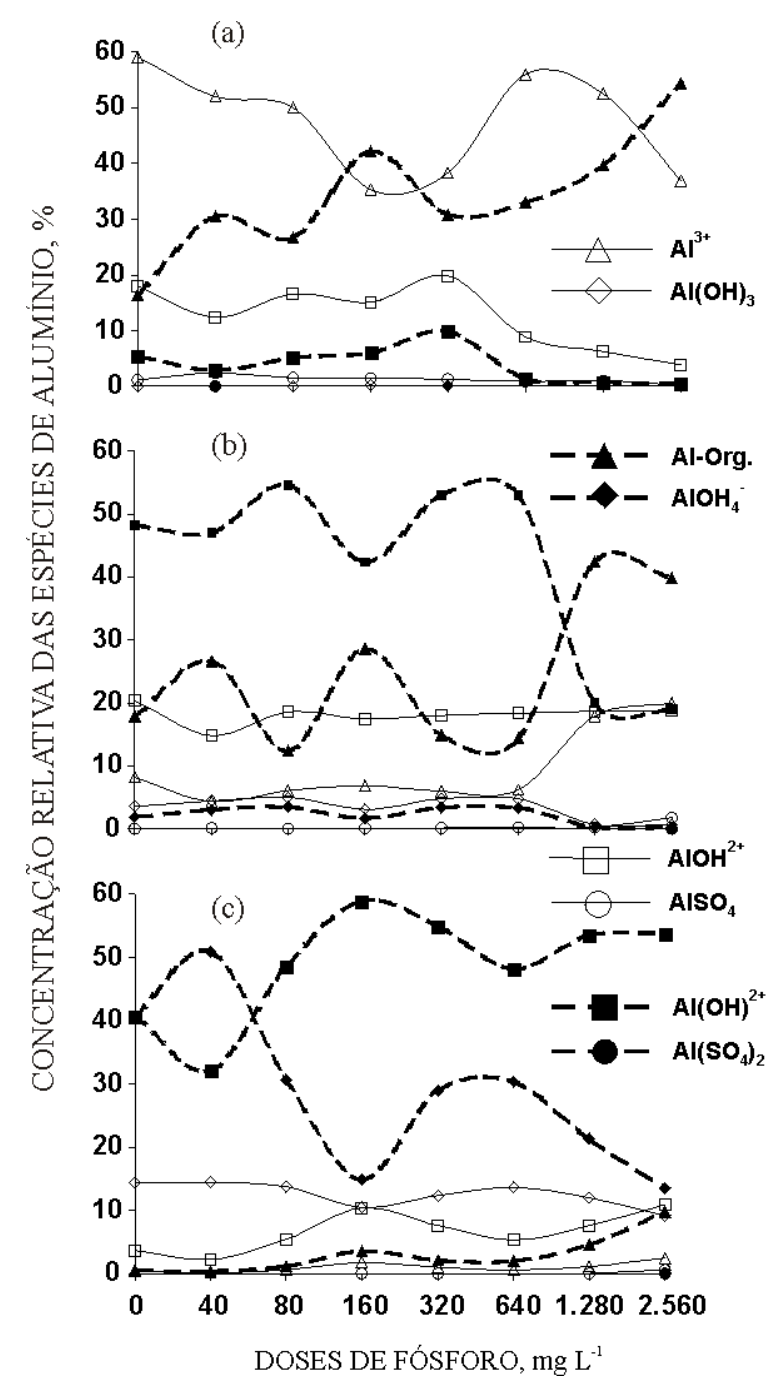

Figura 5. Concentração relativa de espécies de alumínio da solução de um Latossolo Vermelho distrófico típico, cultivado, por sete anos, no sistema plantio direto em diferentes condições de acidez: pH 4,0 (a); pH 4,5 (b) e pH 6,4 (c) e submetidas a aplicação de doses de fósforo. 
(Figura 1b), o que contribuiu para a redução da atividade de $\mathrm{Al}$ em solução (Figura 1c). Isso parece demonstrar a eficiência dos ânions inorgânicos $\left(\mathrm{OH}^{-}\right)$, deslocados do complexo de troca por ação de massa dos fosfatos (Parfitt, 1978 e Novais \& Smyth,1999) (Figura 3c), na complexação do $\mathrm{Al}$ (Al ${ }^{3+}$ livre), diminuindo sua atividade em solução (Figura 5a).

Com base na atividade e na especiação química dos íons na solução do solo, pode-se inferir que o acúmulo superficial de ligantes no sistema plantio direto, bem como o efeito de deslocamento de ânions orgânicos pela aplicação de doses crescentes de P, foram os principais responsáveis pela redução da atividade do $\mathrm{Al}$ em condições de acidez muito elevada, como observado por Hens \& Merckx (2002). Em condições intermediárias de acidez, a atividade de $\mathrm{Al}$
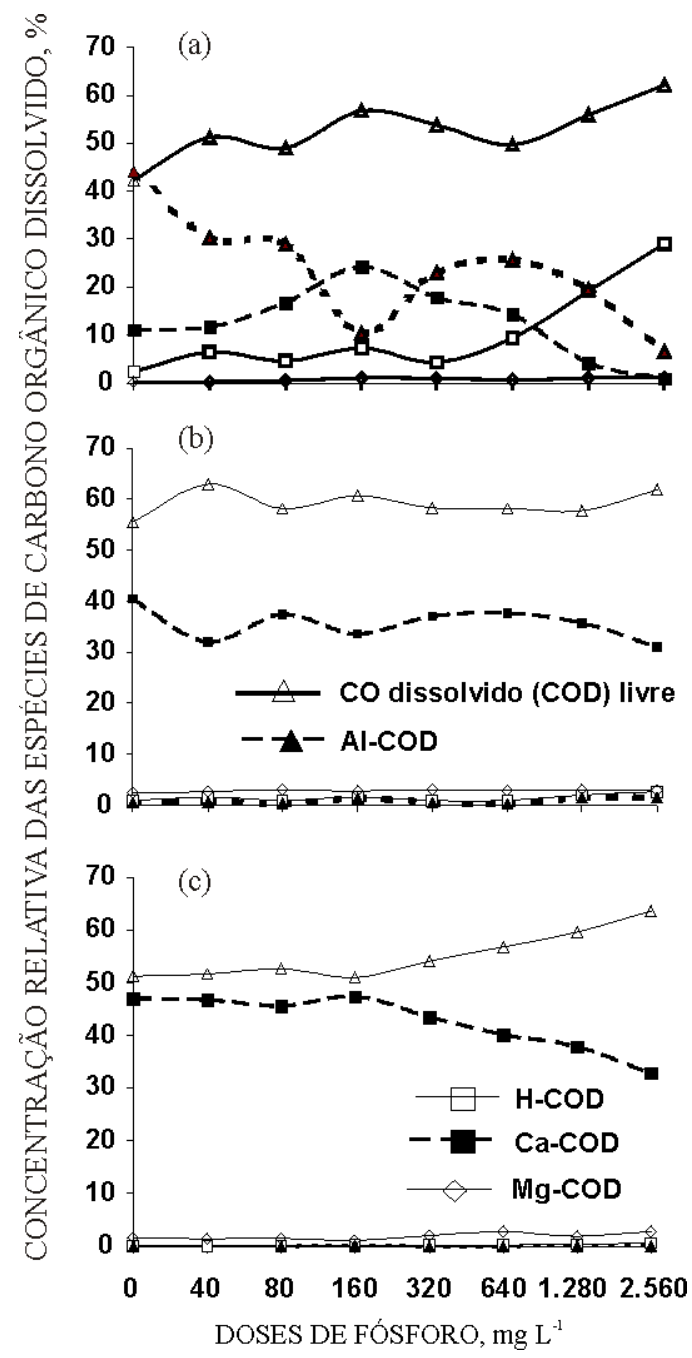

Figura 6. Concentração relativa de espécies de carbono orgânico dissolvido (COD) na solução de um Latossolo Vermelho distrófico típico, cultivado, por sete anos, no sistema plantio direto em diferentes condições de acidez: pH 4,0 (a); pH 4,5 (b) e pH 6,4 (c) e submetidas a aplicação de doses de fósforo. foi muito baixa (Figura 2b), pela ação dos ânions orgânicos e inorgânicos na solução, pois, mesmo após a complexação do $\mathrm{Al}$ (Al-COD), ainda predominava o $\mathrm{CO}$ dissolvido (COD livre). Nessa condição e em $\mathrm{pH}$ mais elevado $(6,4)$, ocorre a formação de Ca-COD, determinada pelo aumento de Ca pela calagem anterior (1994).

\section{CONCL USÕES}

1. Verificou-se a diminuição no teor trocável e na atividade de $\mathrm{Al}$ na solução do solo com o aumento das doses de fosfato aplicadas.

2. Como não houve formação de fosfatos de $\mathrm{Al}$ $\left(\mathrm{AlPO}_{4} ; \mathrm{Al}(\mathrm{OH})_{2} \mathrm{H}_{2} \mathrm{PO}_{4}\right)$ no estudo da especiação iônica, a diminuição da atividade de $\mathrm{Al}$ na solução do solo deveu-se à formação de complexos com outros ânions.

3. Essa complexação, predominantemente na forma de $\mathrm{Al}-\mathrm{CO}$ dissolvido, na condição de maior acidez, e $\mathrm{AlOH}^{2+}$, na condição de acidez intermediária, foi atribuída à liberação de ligantes orgânicos do complexo de troca pela ação do fosfato.

\section{AGRADECIMENTOS}

Os autores agradecem aos pesquisadores da Embrapa-Trigo, Dr. Sírio Wiethölter e Dr. Delmar Pöttker (In Memoriam), pela pronta liberação da área experimental, pelo auxílio e empenho na coleta das amostras de solo e pelas valiosas sugestões ao trabalho.

\section{LITERATURA CITADA}

ALVA, A.K. \& SUMNER, M.E. Alleviation of aluminum toxicity to soybeans by phosphogypsum or calcium sulfate in dilute nutrient solutions. Soil Sci., 147:278-284, 1989.

ALVA, A.K.; EDWARDS, D.G.; ASHER. C.J. \& BLAMEY, F.P.C. Relationships between root lenght of soybean and calculated activities of aluminum monomers in nutrient solutions. Soil Sci. Soc. Am. J., 50:959-962, 1986.

ANGHINONI, I. \& SALET, R.L. Aluminum toxicity in notillage system in Southern Brazil. In: WORLD CONGRESS OF SOIL SCIENCE, 16., Montpellier, 1998. Summaries. Montpellier, International Soil Science Society, 1998. p.261-267

ANGHINONI, I. \& SALET, R.L. Reaplicação de calcário no sistema plantio direto consolidado. In: KAMINSKI, J., ed. Uso de corretivos da acidez do solo no plantio direto. Pelotas, Núcleo Regional Sul, 2000. p.41-59. (Boletim Técnico, 4)

BARROW, N.J. The description of phosphate adsorption curves. J. Soil Sci., 29:447-462, 1978. 
COMISSÃO DE FERTILIDADE DO SOLO - CFSRS/SC. Recomendações de adubação e de calagem para os Estados do Rio Grande do Sul e Santa Catarina. 2.ed. Passo Fundo: Sociedade Brasileira de Ciência do Solo - Núcleo Regional Sul; Embrapa/CNPT, 1989. 128p.

COMISSÃO DE FERTILIDADE DO SOLO - CFSRS/SC. Recomendações de adubação e de calagem para os Estados do Rio Grande do Sul e Santa Catarina. 3.ed. Passo Fundo, Sociedade Brasileira de Ciência do Solo - Núcleo Regional Sul; Embrapa/CNPT, 1995. 225p.

ERNANI, P.R. \& BARBER, S.A. Corn growth and changes of soil and root parameters as affected by phosphate fertilizers and liming. Pesq. Agropec. Bras., 26:1309-1314, 1991.

ERNANI, P.R.; FIGUEIREDO, O.R.A.; BECEGATO, V. \& ALMEIDA, J.A. Decréscimo da retenção de fósforo no solo pelo aumento do pH. R. Bras. Ci. Solo, 20:159-162, 1996.

ERNANI, P.R.; NASCIMENTO, J.A.L.; CAMPOS, M.L. \& CAMILLO, R.J. Influência da combinação de fósforo e calcário no rendimento de milho. R. Bras. Ci. Solo, 24:537$544,2000$.

FRANSON, M.A.H. Phenate method. In: STANDARD methods for examination of water and wastewater. 19.ed. Washington, United Book, 1995. p.480-482

GUSTAFFSON, J.P. Visual Minteq - version 2.12 - 2002 Disponível em: <http://www.lwr.kth.se/enghish/ OurSoftware/Vminteq/index.htm>. Acesso em 01 mar. 2004.

HAYNES, R.J. Lime and phosphate in the soil-plant system. Adv. Agron., 37:249-315, 1984.

HENS, M. \& MERCKX, R. The role of colloidal particles in the speciation and analysis of "dissolved" phosphorus. Water Res., 36:1483-1492, 2002.

HUE, N.V.; CRADDOCK, G.R. \& ADAMS, F. Effects of organic acids on aluminum toxicity in subsoils. Soil Sci. Soc. Am. J., 50:28-34, 1986.

JEFFERY, G.H.; BASSETT, J.; MENDHAM, J. \& DENNEY, R.C. Análise química quantitativa. Rio de Janeiro, Afiliada, 1992. 712p.

LOVATO, T. Dinâmica do carbono e nitrogênio do solo afetado por preparo do solo, sistemas de cultura e adubo nitrogenado. Porto Alegre, Universidade Federal do Rio Grande do Sul, 2001. 133p. (Tese de Doutorado)
MARSCHNER, H. Mineral nutrition of higher plants. 2.ed. San Diego, Academic Press, 1995. 889p.

MENDEZ, J. \& KAMPRATH, E.J. Liming of Latosols and the effect on phosphorus response. Soil Sci. Soc. Am. J., 52:8688, 1978.

MOORE, T.R. The spectrophotometric determination of dissolved organic carbon in peat waters. Soil Sci. Soc. Am. J., 49:1590-1592, 1985.

MURPHY, J. \& RILLEY, J.P. A modified single solution method for the determination of phosphate in natural waters. Anal. Chim. Acta, 27:31-36, 1962.

NOLLA, A. Critérios para a calagem no sistema plantio direto. Porto Alegre, Universidade Federal do Rio Grande do Sul, 2003. 169p. (Tese de Doutorado)

NOVAIS, R.F. \& SMYTH, T.J. Fósforo em solo e planta em condições tropicais. Viçosa, MG, Universidade Federal de Viçosa, 1999. 399p.

PARFITT, R.L. Anion adsorption by soils and soil material. Adv. Agron., 30:1-50, 1978.

RAIJ, B.van. Fertilidade do solo e adubação. Piracicaba, Ceres, 1991. 344p.

RHEINHEIMER, D.S.; CONTE, E. \& ANGHINONI, I. Sorção de fósforo em função do teor inicial e de sistemas de manejo do solo. R. Bras. Ci. Solo, 27:41-49, 2003.

RITCHIE, G.S.P.; NELSON, M.P. \& WHITTEN, M.G. The estimation of free aluminum and the complexation between fluoride and humate anions for aluminum. Comm. Soil Sci. Plant Anal., 19:857-871, 1988.

SALET, R.L.; ANGHINONI, I. \& KOCHHANN, R.A. Atividade do alumínio na solução de solo do sistema plantio direto. R. Ci.. Unicruz, 1:9-13, 1999.

SHOJI, S. \& FUJIWARA, Y. Active aluminum and iron in the humus horizons of Andosols from Northeastern Japan: Their forms, properties, and significance in clay weathering. Soil Sci., 137:216-226, 1984.

SPOSITO, G. The chemistry of soils. New York, Oxford University Press, 1989. 277p.

TEDESCO, M.J.; GIANELLO, C.; BISSANI, C.A.; BOHNEN, H. \& VOLKWEISS, S.J. Análise de solo, plantas e outros materiais. 2.ed. Porto Alegre, Universidade Federal do Rio Grande do Sul, 1995. 174p. (Boletim Técnico, 5)

WRIGHT, R.J.; BALIGAR, V.C.; BELESKY, J.D. \& SNUFFER, J.D. The effect of phosphate rock dissolution on soil chemical properties and wheat seedling root elongation. Plant Soil, 134:21-30, 1991. 